\title{
Acúmulo de metais pesados e alterações químicas em Cambissolo cultivado com meloeiro
}

\author{
Alessandra M. S. Mendes ${ }^{1}$, G ustavo P. Duda ${ }^{2}$, Clistenes W. A. do Nascimento ${ }^{3}$, José Alexsandro G. Lima ${ }^{4} \&$ Aluízio D. L. Medeiros ${ }^{5}$
}

\section{RESU MO}

Com a utilização intensiva de agroquímicos, os solos cultivados podem ter o teor de metais pesados disponíveis às plantas aumentado com possível contaminação subsequente da cadeia trófica. Propôs-se, no presente trabalho, avaliar a influência do tempo de uso agrícola de um Cambissolo Háplico cultivado com meloeiro (Cucumis melo L.) sob o acúmulo de metais pesados e as características químicas do solo. 0 solo com plantios de meloeiro com um, dois e três anos de implantação foram avaliados, além de uma área de vegetação natural, nas camadas de 0-20 e 20-40 cm de profundidade. 0 s resultados mostraram que o tempo de cultivo alterou as características químicas do solo, com aumento do pH e do teor de N, P e K nas duas camadas de solo estudadas; já os teores de Ca foram diminuídos pelo cultivo. Houve acréscimos no teor total e disponível de $\mathrm{Ni}, \mathrm{Pb}, \mathrm{Cu}$ e $\mathrm{Mn}$, na camada superficial, em função do tempo de cultivo do solo, enquanto os teores totais de Fe e $\mathrm{Zn}$ foram reduzidos em ambas as camadas avaliadas, no entanto, os teores disponíveis ainda são relativamente baixos.

Palavras-chave: elementos traços, biodisponibilidade de metais, contaminação de solos

\section{Accumulation of heavy metals and chemical alterations in a Ultisol cultivated with melon}

\begin{abstract}
Soil cultivation using agrochemicals may increase the concentration of availability of heavy metals to plant and cause contamination of the trophic chain as well. This work was carried out to evaluate the influence of length of time under cultivation with melon (Cucumis melo L.) on the heavy metals accumulation and chemical alterations in Ultisol. M elon plantations of one, two and three years were studied. A benchmark area under natural vegetation was used to compare the data obtained. The results showed that the lengthiest time under cultivation altered the chemical characteristics of the soil. An increase in $\mathrm{pH}$ and contents of $\mathrm{N}, \mathrm{P}$ and $\mathrm{K}$ was observed for the two soil layers evaluated. $\mathrm{O}$ the other hand, $\mathrm{Ca}$ contents were decreased by cultivation. The larger the cultivation time the higher the concentration of $\mathrm{N}, \mathrm{Pb}$, $\mathrm{Cu}$, and $\mathrm{Mn}$ in the soil surface layer, whereas the Fe and $\mathrm{Zn}$ concentrations were diminished in both analyzed soil layers, though available concentrations are still regarded as low in soil.
\end{abstract}

Key words: trace elements, metal availability, contamination of soil

\footnotetext{
${ }^{1}$ Embrapa Semiárido, km 156, BR 428, CP 23, CEP 56302-970, Petrolina-PE. Fone: (87) 3862-1711. E-mail: amendes@cpatsa.embrapa.br

2 UAG/U FRPE, Av. Bom Pastor, s/n, Boa Vista, CEP 55296-901, Garanhuns, PE. Fone: (87) 3761-0882. E-mail: gpduda@uag.ufrpe.br

3 DEPA/UFRPE, CEP 52171-900, Recife-PE. E-mail: clistenes@pq.cnpq.br. Fone: (81) 3320-6236. Pesquisador do CN Pq

4 INCRA, CEP 60410-410, Fortaleza, CE. Fone: (85) 3455-9223. E-mail: jose.lima@mba.gov.br

5 Secretaria Municipal de Agricultura, CEP 59695-000, Baraúna, RN. E-mail: adlmce@yahoo.com.br
} 


\section{INTRODUÇÃO}

As atividades antropogênicas, como mineração, disposição de resíduos tóxicos e práticas agrícolas, têm contribuído para o aumento da concentração de metais pesados em solos e, consequentemente, para os riscos da contaminação da cadeia trófica via absorção pelas plantas. $\mathrm{O}$ cultivo intensivo dos solos com utilização de insumos químicos (fertilizantes e pesticidas) pode aumentar o teor de metais pesados disponíveis às culturas.

Vários trabalhos têm demonstrado a presença de concentrações consideráveis de metais pesados em fertilizantes minerais e corretivos de acidez de solos (Alves, 2003; Campos et al., 2005; Mendes et al., 2006). A aplicação de esterco de origem animal, dependendo de sua origem, também pode incorporar metais pesados ao solo provenientes sobretudo dos aditivos usados na alimentação animal (O'Neill, 1993). Ogiyama et al. (2005), por exemplo, relatam que a adubação com esterco de suíno ocasionou aumento no teor de metais pesados no solo, em especial de $\mathrm{Cu}$ e $\mathrm{Zn}$.

A incorporação de metais aos solos tem sido observada tanto em áreas agrícolas próximas a regiões industriais como em regiões em que ocorre exploração agrícola intensiva, utilizando-se alto aporte tecnológico (Ramalho et al., 2000; Mashi et al., 2004; Doelsch et al. 2006; Pruvot, 2006; Banat et al., 2007). Estudos desenvolvidos no Brasil em Argissolo vermelho-amarelo, indicaram a possibilidade de contaminação do sistema solo-água-planta com $\mathrm{Pb}$ e Zn, após aplicação de formulação N-P-K + Zn $(2-28-8+0,5 \% \mathrm{Zn})$ ou do corretivo calcário-resíduo de Paracatu (Amaral Sobrinho et al., 1994). Fernandes et al. (2007) encontraram teor de $\mathrm{Cd}, \mathrm{Cr}$ e $\mathrm{Ni}$ superiores aos valores de intervenção agrícola preconizados pela CETESB (2005) em solos cultivados com olerícolas, no Estado de Minas Gerais.

Os metais pesados incorporados por insumos agrícolas se acumulam principalmente na camada superficial do solo, o que os torna potencialmente disponíveis à absorção pelas plantas, situação que se agrava quando a quantidade de metal acumulada excede a capacidade de retenção do solo, tornando-os facilmente lixiviáveis, com consequente contaminação de águas subterrâneas (Stigliani, 1988); portanto, o monitoramento dos teores de metais pesados disponíveis em áreas com uso intensivo de agroquímicos, é essencial para a sustentabilidade agrícola e para a segurança alimentar.

Nas últimas décadas, o Estado do Rio Grande do Norte tem apresentado grande crescimento na área cultivada com frutas tropicais, passando a ser grande exportador de manga, acerola, banana, mamão e, principalmente, de melão. Entre os solos predominantes nas regiões produtoras se destacam os Cambissolos Eutróficos, originados do calcário que, em geral, apresentam valores de $\mathrm{pH}$ próximos à alcalinidade; devido à baixa disponibilidade de micronutrientes e fósforo, faz-se necessário a aplicação de quantidades elevadas de adubos contendo esses nutrientes, o que pode adicionar, ao solo, os metais. Tal adição, no entanto, pode tornar-se importante quando se considera que a adubação é feita por ciclo de cultivo do meloeiro, possível de acarretar efeito residual de metais pesados (Webb et al., 2001).
Considerando-se os poucos trabalhos relativos a tempo de cultivo e acúmulo de metais pesados no Brasil objetivou-se, no presente trabalho, avaliar a influência do tempo de uso agrícola de um Cambissolo Háplico cultivado com meloeiro (Cucumis melo L.) sob teor de metais pesados acumulados e as características químicas do solo.

\section{MATERIAL E MÉTODOS}

O estudo foi realizado em áreas da Fazenda Vila Nova, município de Baraúna, cuja produção se destina à fruticultura de exportação. A área em estudo faz parte da região da Chapada do Apodi, onde está situado um dos mais importantes polos de irrigação do Nordeste. Baraúna está localizada na microrregião de Mossoró, na altitude de $94 \mathrm{~m}$, longitude (S) de 5 04' 44" e latitude (W) 37 37' 00”.

De acordo com a classificação de Köppen, o clima predominante na região é do tipo BSw'h', caracterizado por ser muito quente e semiárido, com a estação chuvosa atingindo o outono. Os índices pluviométricos se situam, em média, em torno dos $677 \mathrm{~mm}$ por ano. A temperatura média anual é de $27,6{ }^{\circ} \mathrm{C}$ e a vegetação predominante é de caatinga arbustivo-arbórea (Serhid, 2001).

O município de Baraúna apresenta relevo plano, com existência de pequenas depressões, sendo sua declividade máxima de 3\%; os solos mostram textura uniforme ao longo do perfil, ocorrendo solos de textura média a argilosa, com profundidade de 0,5 a $1,0 \mathrm{~m}$, oscilando de rasos a pouco profundos, associados com o afloramento de rochas calcárias da Formação Jandaíra. O solo estudado foi classificado como Cambissolo Háplico Ta eutrófico típico, textura franco-argilo-arenosa, A moderado, caatinga hiperxerófila, relevo plano (EMBRAPA, 2006; Mota, 2004).

Visando avaliar o efeito do tempo de cultivo sobre o acúmulo e disponibilidade dos metais $\mathrm{Cr}, \mathrm{Cd}, \mathrm{Ni}, \mathrm{Pb}, \mathrm{Zn}, \mathrm{Cu}$, $\mathrm{Fe}$ e $\mathrm{Mn}$ no solo, as áreas selecionadas para o estudo apresentavam três tempos de uso do solo (T1 - um, T2 - dois e T3 - três anos) e uma área referencial sob mata nativa de Caatinga, considerada tempo inicial (T4 - sem cultivo). Em todas as áreas, a cada ano eram realizados três cultivos com meloeiro.

Dentro de cada área, com cerca de 1,2 ha cada, foram retiradas três amostras compostas (10 amostras simples para cada amostra composta), nas camadas de solo de 0-20 e 20-40 cm de profundidade. As amostras foram levadas ao laboratório, secadas ao ar e passadas em peneiras de $2 \mathrm{~mm}$ para obtenção da terra fina secada ao ar (TFSA).

$\mathrm{Na}$ adubação mineral do meloeiro se utilizaram $500 \mathrm{~kg} \mathrm{ha}^{-1}$ de monofosfato de amônio (MAP) em fundação, uma vez por cultivo. Os fertilizantes uréia $\left(150 \mathrm{~kg} \mathrm{ha}^{-1}\right)$ e cloreto de potássio $\left(200 \mathrm{~kg} \mathrm{ha}^{-1}\right)$ foram aplicados via fertirrigação, durante o ciclo da cultura; aplicaram-se ainda $20 \mathrm{~m}^{3} \mathrm{ha}^{-1}$ de esterco bovino em fundação. Este procedimento foi repetido em cada ciclo de cultivo.

Foram determinados, nas amostras coletadas, $\mathrm{pH}$ em água, cátions trocáveis $(\mathrm{Ca}, \mathrm{Mg}, \mathrm{K}$ e $\mathrm{Na}), \mathrm{P}$ disponível, $\mathrm{N}$ total, $\mathrm{NO}_{3}{ }^{-}$e carbono orgânico do solo, conforme metodologia da 
EMBRAPA (1999). O teor disponível dos metais pesados foi estimado usando-se o extrator DTPA (Lindsay \& Norvell, 1978), enquanto o teor total foi determinado após digestão com água régia (McGrath \& Cunliffe, 1985). Todas as análises foram realizadas em triplicata com determinação dos metais por espectrofotometria de absorção atômica.

Os dados dos teores de metais pesados foram submetidos a análise de variância pelo teste $\mathrm{F}$, em delineamento inteiramente casualizado, considerando-se um fatorial 4 × 2 (quatro tempo de uso e duas profundidades), com três repetições. As médias foram comparadas pelo teste de Tukey a 5\% de probabilidade.

\section{RESULTADOS E DISCUSSÃO}

O tempo de cultivo do solo proporcionou ligeiro aumento do $\mathrm{pH}$ do solo e dos teores de $\mathrm{N}$ total e $\mathrm{N}-\mathrm{NO}_{3}{ }^{-}$, duplicou os teores de $\mathrm{K}$ e aumentou, em cerca de nove vezes, o teor de $\mathrm{P}$ disponível. Esses efeitos foram similares para as duas camadas de solo estudadas (Tabela 1). Evidentemente, esses resultados corroboram com a mobilidade dos elementos no perfil do solo, com o $\mathrm{P}$ sendo acumulado em maior quantidade devido à sua mais forte retenção nas partículas minerais (Novais \& Smith, 1999). Exceção a este comportamento geral foi observada para o teor de $\mathrm{Ca}$, que diminuiu com o tempo de cultivo, especialmente na camada de $0-20 \mathrm{~cm}$; este elemento não foi adicionado via fertilizantes minerais ou calagem, razão pela qual a absorção pela cultura conduziu a decréscimos na sua concentração no solo; comportamento semelhante pode ser observado para o $\mathrm{Mg}$.

Nota-se, para o carbono orgânico $(\mathrm{C})$, que na camada de 0-20 cm os teores diminuíram consideravelmente quando se compara o solo sob vegetação nativa com o cultivado durante dois anos (Tabela 1); este decréscimo correspondeu a $89 \%$ do teor de C inicial. A diminuição dos teores de C em solos após início de cultivo tem sido observada em diversas áreas (Almeida et al., 2005; Fialho et al., 2006, Silva et al., 2006) e pode ser atribuída à mobilização do solo, o que proporciona maior aeração e decomposição de resídu- os orgânicos; isto é ainda mais importante nas condições estudadas (alta temperatura, irrigação e solo de textura franco-argilo-arenosa), as quais aceleram a decomposição da matéria orgânica. Após três anos de cultivo ocorreu um aumento nos teores de carbono no solo, o que faz supor que as adubações orgânicas utilizadas no período sejam responsáveis por este incremento.

Observa-se, na Tabela 2 , aumento significativo $(\mathrm{P}<0,01)$ nos teores totais de $\mathrm{Pb}$ e $\mathrm{Cu}$ no solo em decorrência do tempo de cultivo; os valores de $\mathrm{Cu}$ no solo com 1 e 2 anos sob cultivo do meloeiro superam o Valor de Prevenção (PV) preconizado pela CETESB (2005) que indica a necessidade do monitoramento ou remediação dos impactos ao ambiente, causados por esses metais, em decorrência de prejuízos à qualidade do solo. $\mathrm{O}$ aporte de $\mathrm{Cu}$ em três anos de cultivo aponta tendência de crescimento ao Valor de Intervenção (VI) para solos agrícolas (200 mg kg-1) (CETESB, 2005), situação na qual medidas de remediação da área devem ser adotadas visando diminuir os riscos à saúde humana. A elevação dos teores totais de $\mathrm{Cu}$ nas áreas pode estar relacionada ao uso de fungicidas cúpricos, prática comum nos plantios de olerícolas, como o meloeiro. Os maiores teores do elemento na camada superficial indicam origem antropogênica e podem estar relacionados, também, com o maior teor de matéria orgânica nesta camada, forte adsorvente para $\mathrm{Cu}$ (Nascimento \& Fontes, 2004).

Os teores de $\mathrm{Pb}$ total no solo (Tabela 2) foram elevados em aproximadamente $70 \%$ em relação aos teores no solo com vegetação nativa. Embora ainda em valores toleráveis, o monitoramento das alterações dos teores desse elemento nas áreas estudadas exige precaução, visto que ultrapassa o valor de referência de qualidade - VRQ (CETESB, 2005), ou seja, são teores anormalmente altos para solos não contaminados.

Ainda em relação à Tabela 2 e embora não significativos estatisticamente, ressalta-se a clara tendência de aumento nas concentrações de $\mathrm{Cr}$ e Ni no solo, o que indica a necessidade de estudos mais detalhados para estabelecer os riscos de contaminação dos produtos cultivados; por outro lado, os teores disponíveis desses elementos avaliados pelo

Tabela 1. Características químicas de um Cambissolo Háplico Ta eutrófico típico, no município de Baraúna, RN, em função do tempo de uso e da profundidade de coleta

\begin{tabular}{|c|c|c|c|c|c|c|c|c|c|}
\hline \multirow{2}{*}{ Tempo de uso } & \multirow[t]{2}{*}{$\mathrm{pH}$} & $\mathrm{Ca}^{2+}$ & $\mathrm{Mg}^{2+}$ & $\mathrm{Na}^{+}$ & $\mathbf{K}^{+}$ & \multirow{2}{*}{$\begin{array}{c}P \\
\left(\mathrm{mg} \mathrm{kg}^{-1}\right)\end{array}$} & \multirow{2}{*}{$\begin{array}{c}\mathrm{NT} \\
\left(\mathrm{g} \mathrm{kg}^{-1}\right)\end{array}$} & \multirow{2}{*}{$\begin{array}{c}\mathrm{NO}_{3}^{-} \\
\left(\mathrm{mg} \mathrm{kg}^{-1}\right)\end{array}$} & \multirow{2}{*}{$\begin{array}{c}\mathrm{C} \\
\left(\mathrm{g} \mathrm{kg}^{-1}\right)\end{array}$} \\
\hline & & \multicolumn{4}{|c|}{$\left(\mathrm{cmol}_{\mathrm{c}} \mathrm{kg}^{-1}\right)$} & & & & \\
\hline & & & & & $0-20 \mathrm{~cm}$ & & & & \\
\hline 0 & 6,67 & 8,73 & 1,53 & 0,08 & 0,49 & 9,44 & 1,33 & 31,89 & 15,87 \\
\hline 1 ano & 6,87 & 8,00 & 2,17 & 0,10 & 0,53 & 12,44 & 1,20 & 26,45 & 2,36 \\
\hline 2 anos & 7,23 & 7,10 & 2,37 & 0,05 & 0,63 & 23,16 & 1,50 & 24,11 & 1,76 \\
\hline 3 anos & 7,53 & 7,20 & 2,00 & 0,09 & 1,10 & 87,16 & 1,60 & 32,67 & 11,15 \\
\hline CV (\%) & 5,40 & 9,84 & 17,62 & 28,16 & 40,66 & 110,59 & 12,58 & 14,47 & 55,99 \\
\hline & & & & & $20-40 \mathrm{~cm}$ & & & & \\
\hline 0 & 6,87 & 7,70 & 1,83 & 0,04 & 0,34 & 7,03 & 1,20 & 40,44 & 4,59 \\
\hline 1 ano & 6,90 & 7,77 & 1,90 & 0,09 & 0,51 & 12,58 & 0,97 & 38,11 & 5,11 \\
\hline 2 anos & 7,13 & 6,97 & 2,10 & 0,06 & 0,45 & 15,64 & 1,27 & 23,33 & 5,37 \\
\hline 3 anos & 7,87 & 7,23 & 2,83 & 0,09 & 0,57 & 77,41 & 1,47 & 32,67 & 6,82 \\
\hline CV $(\%)$ & 6,48 & 5,16 & 21,17 & 33,24 & 20,98 & 117,50 & 16,83 & 22,60 & 17,41 \\
\hline
\end{tabular}

NT - nitrogênio total; C - carbono orgânico 
Tabela 2. Comparação de médias e teste $\mathrm{F}$ para os efeitos dos fatores tempo de uso e profundidade sobre os teores de metais $\left(\mathrm{mg} \mathrm{kg}^{-1}\right)$ extraídos com água régia em um Cambissolo Háplico Ta eutrófico típico, no município de Baraúna, RN

\begin{tabular}{|c|c|c|c|c|c|c|c|c|}
\hline & $\mathrm{Cr}$ & $\mathrm{Cd}$ & $\mathrm{Ni}$ & $\mathrm{Pb}$ & $\mathrm{Zn}$ & $\mathrm{Cu}$ & $\mathrm{Fe}^{(1)}$ & $M n$ \\
\hline \multicolumn{9}{|l|}{ Tempo de uso } \\
\hline 0 & 89,00 & 0,83 & 109,83 & $18,67 \mathrm{~b}$ & 93,00 & $95,17 \mathrm{~b}$ & $13,38 a$ & $280,83 a b$ \\
\hline 1 & 92,67 & 0,17 & 117,33 & $12,00 \mathrm{~b}$ & 88,66 & $102,50 \mathrm{~b}$ & $9,26 a b$ & $273,17 b$ \\
\hline 2 & 116,50 & 0,50 & 139,33 & $17,50 \mathrm{~b}$ & 78,83 & 125,33 a & $6,39 \mathrm{~b}$ & $305,75 a b$ \\
\hline 3 & 152,67 & 0,33 & 169,50 & 31,83 a & 81,33 & $140,50 \mathrm{a}$ & $0,16 \mathrm{c}$ & $431,42 \mathrm{a}$ \\
\hline \multicolumn{9}{|l|}{ Profundidades } \\
\hline $0-20 \mathrm{~cm}$ & 116,58 & 0,41 & 123,33 & 20,92 & 77,42 & $125,92 \mathrm{a}$ & 8,63 & 336,79 \\
\hline $20-40 \mathrm{~cm}$ & 108,83 & 0,50 & 144,67 & 19,08 & 93,50 & $105,83 \mathrm{~b}$ & 5,96 & 308,79 \\
\hline Fatores & \multicolumn{8}{|c|}{$F^{(2)}$} \\
\hline Tempos (T) & $2,08^{\text {ns }}$ & $1,30^{\text {ns }}$ & $2,18^{\text {ns }}$ & $9,42^{* *}$ & $0,68^{\mathrm{ns}}$ & $15,29 * *$ & $14,32 * *$ & $2,02^{\mathrm{ns}}$ \\
\hline Profundidade (P) & $0,14^{\text {ns }}$ & $0,11^{\mathrm{ns}}$ & $1,38^{\text {ns }}$ & $0,45^{\mathrm{ns}}$ & $4,10^{\text {ns }}$ & $14,19 * *$ & $3,30^{\mathrm{ns}}$ & $0,29^{\text {ns }}$ \\
\hline$T \times P$ & $0,47^{\mathrm{ns}}$ & $0,41^{\mathrm{ns}}$ & $0,56^{\mathrm{ns}}$ & $2,71^{\mathrm{ns}}$ & $10,07 * *$ & $3,97^{*}$ & $1,10^{\text {ns }}$ & $1,37^{\mathrm{ns}}$ \\
\hline CV (\%) & 44,16 & 133,61 & 33,15 & 33,56 & 22,75 & 11,27 & 49,25 & 39,34 \\
\hline
\end{tabular}

Médias seguidas da mesma letra na coluna não diferem entre si pelo teste de Tukey a $5 \%$ de probabilidade; ${ }^{(1)} \mathrm{g} \mathrm{kg}^{-1} ;{ }^{1}$ (2) ns, $* *$, * não significativo e significativo a 1 e $5 \%$, respectivamente

DTPA foram muito baixos (Tabela 3) e não representam risco de contaminação da cadeia trófica via absorção pelas plantas.

Foi significativo $(\mathrm{P}<0,01)$ o aumento nos teores de $\mathrm{Ni}$, $\mathrm{Pb}, \mathrm{Zn}$ e $\mathrm{Cu}$ extraídos por DTPA em função do tempo de cultivo do solo (Tabela 3); portanto, os elementos $\mathrm{Pb}$ e $\mathrm{Cu}$ aumentaram não apenas seus teores totais (Tabela 2) mas também as quantidades disponíveis para absorção pelas plantas. Deve-se observar, no entanto, que o extrator utilizado (DTPA) extrai apenas os metais na solução do solo e os facilmente dessorvidos das partículas de solo, implicando que em teores mais elevados de metais podem tornar-se disponíveis em condições de acidificação desses solos de $\mathrm{pH}$ elevado (Tabela 1) ocasionados, por exemplo, por acidificação da rizosfera ou aplicação de fertilizantes amoniacais; deu-se um ligeiro decréscimo dos teores de Fe disponível com o tempo de cultivo que, juntamente com o $\mathrm{Mn}$, se apresenta em teores adequados para o suprimento de plantas. Entre os metais estudados o Mn apresentou os maiores teores biodisponíveis, em concordância com outros trabalhos (Ramalho et al., 2000; Oliveira \& Nascimento, 2006).

Em relação ao fator profundidade, houve efeito significa- tivo $(\mathrm{P}<0,01)$ para os teores de $\mathrm{Cd}, \mathrm{Ni}, \mathrm{Zn}, \mathrm{Cu}$ e $\mathrm{Fe}$, sendo os maiores teores observados na camada superficial $(0-20 \mathrm{~cm})$ do solo. O esterco, dependendo de sua origem, pode conter metais pesados provenientes principalmente dos aditivos usados na alimentação animal (Alloway, 1990; O'Neill, 1993; Nicholson et al., 2006; Li et al., 2006; 2007).

Além dos fertilizantes minerais, a água é outra fonte de contaminação que deve ser considerada em áreas irrigadas na região desse estudo. Salviano et al. (2005), realizando um trabalho de monitoramento da qualidade da água subterrânea utilizada para irrigação nessa região do $\mathrm{RN}$, encontraram que 81 e $100 \%$ das amostras de água analisadas apresentaram, respectivamente, teores de $\mathrm{Cd}$ e $\mathrm{Pb}$ acima dos limites máximos permitidos pela Resolução 20/86 do CONAMA para águas destinadas ao consumo humano e para irrigação de hortaliças e frutíferas e 59 e $100 \%$ das mesmas apresentaram teor de $\mathrm{Cd}$ e $\mathrm{Pb}$ superiores aos valores de intervenção definidos pela CETESB (2005). Os autores concluíram que podem ser adicionados ao solo, por ciclo da cultura por hectare, até $0,168 \mathrm{~kg}$ de $\mathrm{Cd}, 19,348 \mathrm{~kg}$ de $\mathrm{Cu}, 2,348 \mathrm{~kg}$ de $\mathrm{Fe}, 0,193 \mathrm{~kg}$ de $\mathrm{Mn}, 0,553 \mathrm{~kg}$ de $\mathrm{Pb}$ e $14,918 \mathrm{~kg}$ de $\mathrm{Zn}$.

Tabela 3. Comparação de médias e teste $F$ para os efeitos dos fatores tempo de uso e profundidade sobre os teores de metais $\left(\mathrm{mg} \mathrm{kg}^{-1}\right)$ extraídos com DTPA-TEA em um Cambissolo Háplico Ta eutrófico típico, no município de Baraúna, RN

\begin{tabular}{|c|c|c|c|c|c|c|c|c|}
\hline & $\mathrm{Cr}$ & $\mathrm{Cd}$ & $\overline{\mathrm{Ni}}$ & $\mathrm{Pb}$ & $\mathrm{Zn}$ & $\mathrm{Cu}$ & $\mathrm{Fe}^{(1)}$ & $\mathrm{Mn}$ \\
\hline \multicolumn{9}{|l|}{ Tempo de uso } \\
\hline 0 & 0,06 & 0,03 & $0,36 \mathrm{~b}$ & $1,29 \mathrm{~b}$ & $1,34 \mathrm{c}$ & $1,96 \mathrm{c}$ & $8,52 b$ & 59,37 \\
\hline 1 & 0,06 & 0,02 & $0,37 \mathrm{~b}$ & $1,32 \mathrm{~b}$ & $1,38 \mathrm{C}$ & $2,24 b c$ & $9,92 \mathrm{a}$ & 64,67 \\
\hline 2 & 0,05 & 0,03 & $0,41 b$ & $1,61 \mathrm{a}$ & $1,75 \mathrm{~b}$ & $2,61 a b$ & $7,23 \mathrm{c}$ & 66,83 \\
\hline 3 & 0,06 & 0,03 & $0,50 \mathrm{a}$ & $1,70 \mathrm{a}$ & $2,46 \mathrm{a}$ & $2,91 \mathrm{a}$ & $6,88 \mathrm{c}$ & 69,17 \\
\hline \multicolumn{9}{|l|}{ Profundidades } \\
\hline $0-20 \mathrm{~cm}$ & 0,06 & $0,04 \mathrm{a}$ & $0,44 a$ & 1,50 & $1,92 \mathrm{a}$ & $2,65 \mathrm{a}$ & $7,72 b$ & 65,33 \\
\hline $20-40 \mathrm{~cm}$ & 0,06 & $0,02 \mathrm{~b}$ & $0,38 \mathrm{~b}$ & 1,45 & $1,54 \mathrm{~b}$ & $2,21 \mathrm{~b}$ & $8,55 \mathrm{a}$ & 64,68 \\
\hline Fatores & \multicolumn{8}{|c|}{$F^{(2)}$} \\
\hline Tempos (T) & $0,89^{\text {ns }}$ & $1,58^{\mathrm{ns}}$ & $17,20 * *$ & $12,34 * *$ & $52,59 * *$ & $9,15^{* *}$ & $61,73^{* *}$ & $1,95^{\text {ns }}$ \\
\hline Profundidade (P) & $1,33^{\text {ns }}$ & $12,25^{* *}$ & $12,64 * *$ & $0,90^{\text {ns }}$ & $28,28 * *$ & $10,29 * *$ & $22,43^{* *}$ & $0,05^{\text {ns }}$ \\
\hline$T \times P$ & $2,22^{\mathrm{ns}}$ & $0,92^{\mathrm{ns}}$ & $4,13^{*}$ & $1,75^{\text {ns }}$ & $5,86 * *$ & $4,95 *$ & $12,42^{* *}$ & $3,60 *$ \\
\hline CV (\%) & 12,48 & 27,99 & 9,43 & 9,63 & 10,17 & 13,93 & 5,30 & 11,29 \\
\hline
\end{tabular}

Médias seguidas da mesma letra na coluna não diferem entre si pelo teste de Tukey a $5 \%$ de probabilidade; ${ }^{(1)} \mathrm{g} \mathrm{kg}^{-1}{ }^{1}{ }^{(2)}$ ns, ${ }^{* *}$, * não significativo e significativo a 1 e $5 \%$, respectivamente 


\section{CONCLUSÕES}

1. O tempo de cultivo provocou alterações em características químicas do solo, com aumento do $\mathrm{pH}$ e dos teores de $\mathrm{N}$, P e K nas duas camadas de solo estudadas, enquanto os teores de $\mathrm{Ca}$ foram diminuídos pelo cultivo.

2. Houve acréscimos nos teores totais de $\mathrm{Ni}, \mathrm{Pb}, \mathrm{Cu}$ e $\mathrm{Mn}$, na camada superficial em função do tempo de cultivo do solo, enquanto os teores totais de $\mathrm{Fe}$ e $\mathrm{Zn}$ foram reduzidos em ambas as camadas avaliadas.

3. A disponibilidade dos metais $\mathrm{Ni}, \mathrm{Pb}, \mathrm{Zn}, \mathrm{Cu}$ e $\mathrm{Mn}$ foi incrementada em função do cultivo sucessivo do solo; no entanto os teores disponíveis são baixos, mas dentro dos limites de tolerância.

\section{LITERATURA CITADA}

Alloway, B. J The origin of heavy metals in soils. In: Alloway, B. J. Heavy metals in soils. New York: John Wiley \& Sons, 1990. p.29-39.

Almeida, J. A.; Bertol, I.; Leite, D.; Amaral, A. J.; Zoldan Júnior, W. A. Propriedades químicas de um Cambissolo Húmico sob preparo convencional e semeadura direta após seis anos de cultivo. Revista Brasileira de Ciência do Solo, v.29, n.3, p.437-445, 2005.

Alves, R. N. Avaliação de um fosfato natural e termofosfatos quanto aos teores totais de metais pesados e à disponibilidade de micronutrientes. Viçosa: UFV, 2003. 35p. Dissertação Mestrado

Amaral Sobrinho, N. M. B.; Velloso, A. C. X.; Costa, L. M.; Oliveira, C. de. Formas químicas de zinco e sua absorção por plantas de milho cultivadas em solo tratado com resíduo siderúrgico. Revista Brasileira de Ciência do Solo, v.18, n.1, p.313-320, 1994.

Banat, K.M.; Howari, M.; To'mah, M.M. Chemical fractionation and heavy metal distribution in agricultural soils, north of Jordan valley. Soil \& Sediment Contamination, v.16, n.1, p.89-107, 2007.

Campos, M. L.; Silva, F. N.; Furtini Neto, A. E.; Guilherme, L. R. G.; Marques, J. J.; Antunes, A. S. Determinação de cádmio, cobre, cromo, níquel, chumbo e zinco em fosfatos de rocha. Pesquisa Agropecuária Brasileira, v.40, n.4, p.361-367, 2005.

CETESB - Companhia de Tecnologia de Saneamento Ambiental. Relatório de estabelecimento de valores orientadores para solos e águas subterrâneas no Estado de São Paulo. São Paulo: Secretaria de Estado do Meio Ambiente, 2005.

Doelsch, E.; Kerchove,V. van de; Saint Macary, H. S. Heavy metal content in soils of Reunion (Indian Ocean). Geoderma, v.34, n.1-2, p.119-134, 2006.

EMBRAPA - Empresa Brasileira de Pesquisa Agropecuária. Manual de análises químicas de solos, plantas e fertilizantes. Rio de Janeiro: Embrapa-CNPS, 1999. 370p.

EMBRAPA - Empresa Brasileira de Pesquisa Agropecuária. Sistema Brasileiro de Classificação de Solos. Rio de Janeiro: Embrapa-CNPS, 2006. 412p
Fernandes, R. B. A.; Luz, W. V.; Fontes, M. P. F.; Fontes, L. E. F. Avaliação das concentrações de metais pesados em áreas olerícolas no Estado de Minas Gerais. Revista Brasileira de Engenharia Agrícola e Ambiental, v.11, n.1, p.81-93, 2007.

Fialho, J. S.; Gomes, V. F. F.; Oliveira, T. S.; Silva Júnior, J. M. T. Indicadores da qualidade do solo em áreas sob vegetação natural e cultivo de bananeiras na Chapada do Apodi-CE. Revista Ciência Agronômica, v.37, n.3, p.250-257, 2006.

Li, S.; Liu, R. L.; Wang, M.; Wang, X. B.; Shan, H.; Wang, H.T. Phytoavailability of cadmium to cherry-red radish in soils applied composted chicken or pig manure. Geoderma, v.136, n.1-2, p.260-271, 2006.

Li, Z. G.; Bian, C. Z.; Jie, X. L. Characteristic of Cd sorption in the copper tailings wasteland soil by amended dissolved organic matter from fresh manure and manure compost. African Journal of Biotechnology, v.6, n.3, p.227-234, 2007.

Lindsay, W. L., Norvell, W. A. Development of a DTPA soil test for zinc, iron, manganese, and copper. Soil Science Society of American Journal, v.42, p.421-428, 1978.

Mashi, S. A.; Yaro, S. A.; Haiba, A. S. Cu, Mn, Fe, and Zn levels in soils of Shika area. Biomedical and Environmental Sciences, v.17, n.4, p.426-431, 2004.

McGrath, S. P.; Cunliffe, C. H. A simplified method for the extraction of metals $\mathrm{Fe}, \mathrm{Zn}, \mathrm{Cu}, \mathrm{Ni}, \mathrm{Cd}, \mathrm{Pb}, \mathrm{Cr}, \mathrm{Co}$ and $\mathrm{Mn}$ from soils and sewage sludges. Journal of Science of Food and Agriculture, v.36, p.794-798, 1985.

Mendes, A. M. S.; Duda, G. P.; Nascimento, C. W. A. do; Silva, M. O. Bioavailability of cadmium and lead in a soil amended with phosphorus fertilizers. Scientia Agricola, v.63, n.4, p.328-332, 2006.

Mota, J. C. A. Caracterização física, química e mineralógica, como suporte para o manejo, dos principais solos explorados com melão na Chapada do Apodi, RN. Fortaleza: UFC, 2004. 96p. Dissertação Mestrado

Nascimento, C. W. A.; Fontes, R. L. F. Correlação entre características de Latossolos e parâmetros de equações de adsorção de cobre e zinco. Revista Brasileira de Ciência do Solo, v.28, n.6, p.965-971, 2004.

Nicholson, F. A.; Mciwem, S. R. S.; Alloway, B. J.; CarltonSmith, C.; Chambers, B. J. Quantifying heavy metal inputs to agricultural soils in England and Wales. Water and Environment Journal, v.20, n.2, p.87-95, 2006.

Novais, R. F.; Smyth, T. J. Fósforo em solo e planta em condições tropicais. Viçosa: DPS/UFV, 1999. 399p.

Ogiyama, S.; Sakamoto, K.; Suzuki, H.; Ushio, S.; Anzai, T.; Inubushi, K. Accumulation of zinc and copper in an arable field after animal manure. Application Soil Science \& Plant Nutrition, v.51, n.6, p.801-808, 2005.

O'Neill, P. Environmental chemistry. 2.ed., London: Chapman \& Hall. 1993, 267p.

Oliveira, A. B.; Nascimento, C. W. A. Formas de manganês e ferro em solos de referência de Pernambuco. Revista Brasileira de Ciência do Solo, v.30, n.1, p.99-110, 2006.

Pruvot, C.; Douay, F.; Herve, F.; Waterlot, C. Heavy metals in soil, crops and grass as a source of human exposure in the former mining areas. Journal of Soils and Sediments, v.6, n.4, p.215-220, 2006. 
Ramalho, J. F. G. P.; Amaral Sobrinho, N. M. B.; Velloso, A. C. $X$. Contaminação da microbacia de Caetés com metais pesados pelo uso de agroquímicos. Pesquisa Agropecuária Brasileira, v.35, n.7, p.1289-1303, 2000.

Salviano, A. M.; Duda, G. P.; Lima, J. A. G.; Holanda, J. S. de; Amorim, L. B.; Paz, K. K. R. da. Teores de metais pesados na água subterrânea utilizada para irrigação no município de Baraúna-RN. In: Congresso Nacional de Irrigação e Drenagem, Simpósio Internacional de Uso das Águas Subterrâneas em Iirrigação. 15, 2005 , Teresina. Anais... Teresina: ABID; Governo do Estado do Piauí; Embrapa meio Norte; DNOCS; CODEVASF, 2005. CD Rom.

Serhid. Bacia 01 - Apodi-Mossoró. http://www.serhid.rn.gov.br// detalhe.asp?IdPublicaçao=137>. 22 Ago. 2001.
Silva, M. A. S.; Mafra, Á. L.; Albuquerque, J. A.; Rosa, J. D.; Bayer, C.; Mielniczuk, J. Propriedades físicas e teor de carbono orgânico de um Argissolo Vermelho sob distintos sistemas de uso e manejo. Revista Brasileira de Ciência do Solo. v.30, n.2 p.329-337, 2006.

Stigliani, W. M. Changes in valued "capacities" of soils and sediments as indicators of nonlinear and time-delayed environmental effects. Environmental Monitoring and Assessment, v.10, p.245-307, 1988.

Webb, J.; Loveland, P. J.; Chambers, B. J.; MItchell, R.; Garwood, T. The impact of modern farming practices on soil fertility and quality in England and Wales. Journal of Agricultural Science, v.137, n.2, p.127-138, 2001. 